\title{
1 Replication and cross-validation of T2D subtypes based on clinical variables: an IMI-
}

\section{RHAPSODY study}

3

4 Roderick C Slieker ${ }^{1,2, *}$, Louise A Donnelly ${ }^{3, *}$, Hugo Fitipaldi ${ }^{4}$, Gerard A Bouland ${ }^{2}$, Giuseppe

5 N. Giordano ${ }^{4}$, Mikael Åkerlund ${ }^{4}$, Mathias J. Gerl ${ }^{5}$, Emma Ahlqvist ${ }^{4}$, Ashfaq Ali ${ }^{6}$, Iulian

6 Dragan $^{7}$, Andreas Festa $^{8,9}$, Michael K. Hansen ${ }^{10}$, Dina Mansour $\mathrm{Aly}^{4}$, Min Kim ${ }^{6,11}$, Dmitry

7 Kuznetsov $^{7}$, Florence Mehl ${ }^{7}$, Christian Klose ${ }^{5}$, Kai Simons ${ }^{5}$, Imre Pavo ${ }^{8}$, Timothy J.

8 Pullen $^{12,13}$, Tommi Suvitaival ${ }^{6}$, Asger Wretlind ${ }^{6}$, Peter Rossing ${ }^{6}$, Valeriya Lyssenko ${ }^{14,15}$,

9 Cristina Legido Quigley ${ }^{6,11}$, Leif Groop ${ }^{4,16}$, Bernard Thorens ${ }^{17}$, Paul W Franks ${ }^{4,18}$, Mark

10 Ibberson $^{7}$, Guy A Rutter ${ }^{13,19}$, Joline WJ Beulens ${ }^{1,20}$, Leen M 't Hart ${ }^{1,2,21, *}{ }^{*}$, Ewan R

11 Pearson $^{3, *, \dagger \dagger}$

${ }^{1}$ Department of Epidemiology and Biostatistics, Amsterdam Public Health Institute,

14 Amsterdam UMC, location VUMC, Amsterdam, the Netherlands

${ }^{2}$ Department of Cell and Chemical Biology, Leiden University Medical Center, Leiden, the

16 Netherlands

${ }^{3}$ Population Health \& Genomics, School of Medicine, University of Dundee, Dundee, UK.

${ }^{4}$ Genetic and Molecular Epidemiology Unit, Lund University Diabetes Centre, Department of

19 Clinical Sciences, CRC, Lund University, SUS, Malmö, Sweden

${ }^{6}$ Steno Diabetes Center Copenhagen, Gentofte, Denmark

${ }^{7}$ Vital-IT Group, SIB Swiss Institute of Bioinformatics, Lausanne, Switzerland

${ }^{8}$ Eli Lilly Regional Operations GmbH, Vienna, Austria.

${ }^{9} 1$ st Medical Department, LK Stockerau, Niederösterreich, Austria.

${ }_{10}$ Cardiovascular and Metabolic Disease Research, Janssen Research \& Development, Spring House, Pennsylvania, U.S.A.

${ }^{11}$ Institute of Pharmaceutical Science, Faculty of Life Sciences and Medicines, King's College London, London, United Kingdom and Metabolism, Department of Metabolism, Digestion and Reproduction, Imperial College London, London, U.K. 
medRxiv preprint doi: https://doi.org/10.1101/2020.12.21.20248628; this version posted December 22, 2020. The copyright holder for this preprint (which was not certified by peer review) is the author/funder, who has granted medRxiv a license to display the preprint in perpetuity.

It is made available under a CC-BY 4.0 International license .

$35{ }^{15}$ Genomics, Diabetes and Endocrinology Unit, Department of Clinical Sciences Malmö,

36 Lund University Diabetes Centre, Skåne University Hospital, Malmö, Swede

$37{ }^{16}$ Finnish Institute of Molecular Medicine, Helsinki University, Helsinki, Finland

$38{ }^{17}$ Center for Integrative Genomics, University of Lausanne, CH-1015, Lausanne, Switzerland

$39{ }^{18}$ Department of Nutrition, Harvard School of Public Health, Boston, MA, USA

$40{ }^{19}$ Lee Kong Chian School of Medicine, Nan Yang Technological University, Singapore

$41{ }^{20}$ Julius Center for Health Sciences and Primary Care, University Medical Center Utrecht,

42 Utrecht, the Netherlands

$43{ }^{21}$ Department of Biomedical Data Sciences, Section Molecular Epidemiology, Leiden

44 University Medical Center, Leiden, the Netherlands

$45 *$ Contributed equally

46

47 Corresponding authors

$48{ }^{\dagger}$ Leen 't Hart, $\mathrm{PhD}$

49 Einthovenweg 20

50 2333ZC Leiden

51 The Netherlands

52 Telephone: +31 715269796

53 E-mail: 1mthart@lumc.nl

54

$55{ }^{\dagger \dagger}$ Prof Ewan Pearson, MD

56 Ninewells Hospital

57 Dundee

58 DD1 9SY

59 United Kingdom

60 Telephone: +44 1382383387

61 E-mail: E.Z.Pearson@ dundee.ac.uk

62

63 Word count: 1708 
64

65

66

67

68

69

70

71

72

73

74

75

76

77

78

79

80

81

82

83

84

85

86

87

88

91

92

93

94

95

96

97

\section{ABSTRACT}

\section{Aims/hypothesis}

Five clusters based on clinical characteristics have been suggested as diabetes subtypes: one autoimmune and four subtypes of type 2 diabetes (T2D). In the current study we replicate and cross-validate these T2D clusters in three large cohorts using readily measured variables in the clinic.

\section{Methods}

In this cross-sectional study, 15,940 individuals were clustered based on age, BMI, HbA1c, random or fasting C-peptide and HDL in three independent cohorts. Clusters were crossvalidated against the original clusters based on HOMA measures. In addition, between cohorts, clusters were cross-validated by re-assigning people based on each cohort's cluster centres.

\section{Results}

Five distinct T2D clusters were identified and mapped back to the original four ANDIS clusters. Using C-peptide and HDL instead of HOMA-B and HOMA-S three of the clusters mapped with high sensitivity $(80.6-90.7 \%)$ to the previously identified Severe Insulin Deficient (SIDD), Severe insulin resistant (SIRD) and Obese (MOD) clusters. The previously described ANDIS MARD cluster could be mapped to the two milder groups in our study one characterised by high HDL, and the other having not any extreme characteristic (MDH cluster). When these two milder groups were combined they mapped well to the previously labelled MARD cluster (sensitivity 79.4\%). In the cross-validation between cohorts, particularly the SIDD and MDH cluster cross-validated well with sensitivities ranging from $73.3 \%$ to $97.1 \%$. SIRD and MD showed a lower sensitivity ranging from $36.1 \%$ to $92.3 \%$ where individuals shifted from SIRD to MD and vice versa.

\section{Conclusions/interpretation}

Clusters based on C-peptide instead of HOMA measures result in clusters that resemble those based on HOMA measures, especially for SIDD, SIRD and MOD. By adding HDL, the MARD cluster based upon HOMA measures resulted in the current clustering in two clusters with one cluster having high HDL levels. Cross-validation between cohorts showed generally a good resemblance between cohorts. Together, our results show that the clustering based on 
medRxiv preprint doi: https://doi.org/10.1101/2020.12.21.20248628; this version posted December 22, 2020. The copyright holder for this preprint (which was not certified by peer review) is the author/funder, who has granted medRxiv a license to display the preprint in perpetuity.

It is made available under a CC-BY 4.0 International license .

98 clinical variables readily measured in the clinic (age, HbA1c, HDL, BMI and C-peptide)

99 results in informative clusters that are representative of the original ANDIS clusters and stable 100 across cohorts. 
medRxiv preprint doi: https://doi.org/10.1101/2020.12.21.20248628; this version posted December 22, 2020. The copyright holder for this preprint (which was not certified by peer review) is the author/funder, who has granted medRxiv a license to display the preprint in perpetuity.

It is made available under a CC-BY 4.0 International license .

\section{INTRODUCTION}

102 A recent study stratified people with any form of diabetes into five clusters based on six

103 clinical variables, i.e. age, glutamate decarboxylase (GAD) antibodies, BMI, HbA1c, insulin

104 resistance (HOMA2-IR) and $\beta$-cell function estimates (HOMA2-B).[1] The five clusters were

105 characterized by autoimmunity (SAID), insulin deficiency (SIDD), insulin resistance (SIRD),

106 high BMI (MOD) and the last without any extreme characteristics other than high age

107 (MARD).[1] Clustering of people with diabetes has been repeated successfully in several

108 other studies based on these variables in people from European descent, other ethnicities and

109 based on different clinical measures.[2-9] In addition, the original and subsequent papers have

110 shown that people in different clusters have different risks for a number of diabetes related

111 outcomes.[1-4] The autoimmunity and insulin deficient clusters were defined by high HbA1c

112 at diagnosis and had higher risk on ketoacidosis and retinopathy[2, 7] and progressed more

113 rapidly onto insulin relative to the other clusters.[1] Moreover, a recent study comprising

114 multiple cohorts enriched for cardiovascular risk assigned people to the clusters identified by

115 Ahlqvist et al.[1] based on the distance to the respective cluster centres. In this study, people

116 in the SIDD cluster showed higher risk of MACE.[5] For the insulin resistant cluster a higher

117 frequency of non-alcoholic fatty liver disease has been observed and people in this group

118 were at increased risk of developing chronic kidney disease.[1] As HOMA calculations

119 require fasting insulin or c-peptide and fasting glucose their measurement is not routine in

120 clinical practice.

121 The aim of the current study is to perform a systematic replication and cross-validation

122 of clustering based on five routine clinical variables in three large international cohorts (DCS,

123 ANDIS, GoDARTS). In ANDIS, we directly compare the current clustering with those

124 identified in the original study.[1] 


\section{METHODS}

\section{Cohort descriptions}

128 Data from 15,940 individuals from three cohorts, DCS (Netherlands), GoDARTS (Scotland)

129 and ANDIS (Sweden) were used in this cross-sectional study. Inclusion criteria for

130 RHAPSODY were age of diagnosis was $\geq 35$, clinical data available within 2 years after

131 diagnosis, GAD negative, no missing data in one of the five for clustering used clinical

132 measures and the presence of GWAS data.

Hoorn DCS cohort

135 The Hoorn Diabetes Care System (DCS) cohort is an open prospective cohort started in 1998 136 with currently over 14,000 individuals with T2D from the northwest part of the

137 Netherlands.[10] The study has been approved by the Ethical Review Committee of the VU

138 University Medical Center, Amsterdam. People visit DCS annually to monitor their diabetes.

139 During this visit multiple measurements are collected as part of routine care, including

140 anthropometric- and lab measurements. Measurements were used anonymously. Individuals

141 were informed about the use of their data and were offered an opt-out. All laboratory

142 measurements were done on samples taken in a fasted state. HbA1c measurements were

143 performed using the turbidimetric inhibition immunoassay for haemolyzed whole EDTA

144 blood (Cobas c501, Roche Diagnostics, Mannheim, Germany, run CV 1.6\%).[10] HDL

145 (mmol/L) was measured enzymatically (Cobas c501, Roche Diagnostics). C-peptide was

146 measured on a DiaSorin Liaison (DiaSorin, Saluggia, Italy). In total, 2,953 individuals

147 matched the inclusion criteria.

148

149

GoDARTS

150 For clinical purposes, individuals with diabetes mellitus from the Tayside region of Scotland

$151 \quad(\mathrm{~N} \square=\square 391,274$; January 1996) were added to the DARTS register.[11] Retrospective and

152 prospective longitudinal anonymized data were collected, including data on prescribing,

153 biochemistry, and clinical data. All laboratory measurements were measured in a non-fasted

154 state. People with T2D were asked to participate in the Genetics of Diabetes Audit and

155 Research Tayside Study (GoDARTS), which currently includes over 10,000 individuals with

156 T2D.[11] The GoDARTS study was approved by the Tayside Medical Ethics Committee.

157 Informed consent was obtained from all participants. C-peptide was measured on a DiaSorin

158 Liaison (DiaSorin, Saluggia, Italy). In total, 5,509 individuals matched the inclusion criteria. 
161 The All New Diabetics in Scania (ANDIS) cohort aims to recruit all people with incident diabetes within Scania County, Sweden. Recruitment started in January 2008 until November 2016. People are included in the study close to diagnosis, with a median of 40 days (IQR 1299). All laboratory measurements were measured in a fasted state. HbA1c measurements were obtained from the Clinical Chemistry database. C-peptide were determined with an electrochemiluminescence immunoassay on Cobas e411 (Roche Diagnostics, Mannheim, Germany) or a radioimmunoassay (Human C-peptide RIA; Linco, St Charles, MO, USA; or Peninsula Laboratories, Belmont, CA, USA). In total, 7,478 individuals matched the inclusion criteria.

\section{Statistical analysis}

171 Clustering was performed on five risk factors for type 2 diabetes progression [12], age

172 (years), BMI $\left(\mathrm{kg} / \mathrm{m}^{2}\right), \mathrm{HbA} 1 \mathrm{c}(\mathrm{mmol} / \mathrm{mol}), \mathrm{HDL}(\mathrm{mmol} / \mathrm{L})$ and C-peptide (nmol/L). The latter

173 two were included as proxies of beta-cell function and insulin sensitivity in absence of fasting 174 glucose (GoDARTS) and insulin (DCS and GoDARTS) and therefore HOMA measures in 175 these cohorts in GoDARTS. K-means clustering was performed separately for males and 176 females using the kmeansruns function in the R-package $f p c$. The optimal number of clusters 177 was determined using the gap statistic across the three cohorts,[13] this being defined as the 178 point where the curve of the GAP statistic versus the number of clusters flattened, with little 179 added value of increasing the number of clusters. The stability of the clusters was assessed in 180 two ways. The clusters identified here in ANDIS using C-peptide instead of HOMA were 181 compared to their previously published clusters based on HOMA.[1] Second, identified 182 clusters were cross-validated between cohorts to assess their stability. For this, individuals 183 from cohort A were assigned to clusters based on the cluster centres of each of the clusters 184 identified in cohort B. Next, predicted clusters in cohort A based on the clusters of cohort B 185 were compared to the 'real' clusters of cohort A. This was done for each of the three pairwise 186 comparisons (DCS-GoDARTS, DCS-ANDIS, GoDARTS-ANDIS). Agreement between 187 clusters was accessed based on the specificity and sensitivity.

188 Analyses were performed using R statistics (version 3.6.2). Figures were produced using the 189 R-package ggplot2 (v3.3.0) and omicCircos (v1.22.0). 
190

191

192

193

194

195

196

197

198

199

200

201

202

203

204

205

206

207

208

209

210

211

212

213

214

215

216

217

218

219

220

221

222

223

\section{RESULTS}

\section{Clustering in three large cohorts based on clinical measures}

In this cross-sectional study, 15,940 individuals from three cohorts were included for which baseline characteristics are given in Table 1. The characteristic of the three cohorts were generally comparable with the majority males and an average of around sixty years. Individuals were clustered based on age, BMI, HbA1c, C-peptide and HDL. Based on the gap-statistic across the three cohorts, the most optimal number of clusters was five (Fig. 1, Fig. S1a). The first cluster comprised 13-17\% of the individuals included. It was characterized by high HbA1c, but compared to the other clusters, younger with lower BMI, C-peptide and HDL levels. When compared to the original clusters in ANDIS[1], this cluster was most similar to the Severe Insulin-Deficit Diabetes (SIDD) cluster with a sensitivity (SE) of $90.7 \%$ (confidence interval (CI), 88.4-92.6\%, Fig. 1, Fig. S1b).[1] Between 9 and 22\% of individuals clustered to a cluster with high C-peptide levels and age, but relatively lower Hbalc and HDL levels, suggestive of insulin resistance. Indeed, compared to the ANDIS clusters, this cluster resembled most that of the Severe Insulin-Resistant Diabetes cluster (SIRD) with SE of 92.4\% (CI, 89.7-94.6\%, Fig. 1, Fig. S1b).[1] The third cluster was comprised of participants with high BMI and the youngest age and relatively lower levels of HbA1c and HDL. It was most similar to the originally described Mild Obesity-related Diabetes cluster (MOD) with a SE of $80.6 \%$ (CI, 78.4-82.7\%) and comprised 18-23\% of the individuals included in the study. The fourth and fifth clusters were most similar to the Mild Age Related Diabetes cluster and showed a combined sensitivity of $79.1 \%$ (CI, 77.5-80.6\%) against the MARD cluster in ANDIS (Fig. 1, Fig. S1b).[1] The fourth cluster, which was also the largest encompassing $29-35 \%$ of the individuals, showed no extreme characteristics and was termed Mild Diabetes. The fifth cluster was characterized by higher age and HDL and termed Mild Diabetes with high HDL (MDH) and comprised 16-19\% of the individuals, Fig. 1).

\section{Clusters cross-validate between the three cohorts}

To assess the stability across cohorts, clusters were cross-validated between cohorts. Clusters generally cross-validated well between the three cohorts (Fig. S2, Table S1). The SIDD and MDH clusters showed the highest sensitivity of the five clusters identified ranging from $85.6 \%$ (CI, 83.5-87.6\%) to $97.1 \%$ (CI, 94.8-98.5\%) and 73.3\% (CI, 69.5-77.0\%) to $92.9 \%$ (CI, 91.3-94.3\%, Fig. S2, Table S1). The SIRD and the MD cluster performed generally worst in terms of sensitivity, with sensitivities ranging from $36.1 \%$ (CI, 32.3-39.9\%) to $92.3 \%$ (CI, 
224

225

226

227

228

229

230

231

232

233

234

235

236

237

238

239

240

241

242

243

244

245

246

247

248

249

250

251

252

253

254

255

256

257

90.1-94.2\%) in SIRD and 40.8\% (CI, 38.9-42.7\%) to 78.1\% (CI, 75.9-80.2\%). Individuals clustered to SIRD were classified as MD and vice versa (Fig. S2, Table S1). The sensitivity of the MOD cluster ranged from $55.0 \%$ (CI, 52.6-57.3\%) to $93.2 \%$ (CI, 91.5-94.7\%).

\section{DISCUSSION}

Based on five clinical variables, people with type 2 diabetes from three large European cohorts were assigned to five separate clusters. Clusters were successfully cross-validated against the clustering reported by Ahlqvist et al.[1] but also between cohorts included.

Even though we used slightly different parameters for clustering - i.e. C-peptide and HDL instead of HOMA measures [1], people were clustered largely to the same clusters in a direct comparison with previously published clusters in ANDIS. The insulin deficient cluster (SIDD) was defined by a high HbA1c, the insulin resistant by a high C-peptide (SIRD), and the obese cluster by a high BMI (MOD). The previously identified MARD cluster [1], could be further divided into two clusters of people with a low (MD-cluster) and a high HDL (MDH-cluster). Including HDL resulted two clusters with mild characteristics, where one had high HDL and one cluster generally a low HLD. A subset of the SIRD cluster was classified as MD, which is most likely due to the use of C-peptide and HDL instead of HOMA measures.

In addition to a comparison to the original ANDIS clusters, in the current study we also cross-validated the clusters across cohorts. Clusters cross-validated generally well and the best sensitivity was observed in the SIDD and MDH clusters. For SIRD and MD a lower sensitivity was observed. Individuals that were classified in one cohort to SIRD but also MOD, were classified as MD in a second cohort and vice versa. The characteristics of particularly SIRD and MD are very similar with the sole difference higher levels of C-peptide in the SIRD cluster. This could explain the difference in classification in the two cohorts.

\section{CONCLUSION}

In the current study, clusters were identified in three cohorts, based on five different clinical characteristics. We show that clusters based on random or fasted C-peptide instead of HOMA measures result in clusters that resemble those based on HOMA measures. By adding HDL we identified one additional cluster with mild characteristics. Cross-validation between cohorts showed that there was generally a good resemblance between cohorts. Together, our results show that the clustering is generally stable across cohorts, also when the clustering includes C-peptide instead of HOMA measures. 


\section{REFERENCES}

260 [1] Ahlqvist E, Storm P, Karajamaki A, et al. (2018) Novel subgroups of adult-onset

261 diabetes and their association with outcomes: a data-driven cluster analysis of six variables.

262 The Lancet Diabetes \& Endocrinology 6: 361-369

263 [2] Safai N, Ali A, Rossing P, Ridderstråle M (2018) Stratification of type 2 diabetes

264 based on routine clinical markers. Diabetes research and clinical practice 141: 275-283.

265 10.1016/j.diabres.2018.05.014

266 [3] Zaharia OP, Strassburger K, Strom A, et al. (2019) Risk of diabetes-associated

267

268 diseases in subgroups of patients with recent-onset diabetes: a 5-year follow-up study. The lancet Diabetes \& endocrinology 7(9): 684-694. 10.1016/S2213-8587(19)30187-1 [4] Dennis JM, Shields BM, Henley WE, Jones AG, Hattersley ATJTLD, Endocrinology (2019) Disease progression and treatment response in data-driven subgroups of type 2 diabetes compared with models based on simple clinical features: an analysis using clinical trial data. 7(6): 442-451

272

273

[5] Kahkoska AR, Geybels MS, Klein KR, et al. (2020) Validation of distinct type 2

274 diabetes clusters and their association with diabetes complications in the DEVOTE, LEADER and SUSTAIN-6 cardiovascular outcomes trials. Diabetes Obes Metab 22(9): 1537-1547. 10.1111/dom.14063

[6] Zou X, Zhou X, Zhu Z, Ji L (2019) Novel subgroups of patients with adult-onset diabetes in Chinese and US populations. Lancet Diabetes Endocrinol 7(1): 9-11. 10.1016/s2213-8587(18)30316-4

[7] Anjana RM, Baskar V, Nair ATN, et al. (2020) Novel subgroups of type 2 diabetes and their association with microvascular outcomes in an Asian Indian population: a datadriven cluster analysis: the INSPIRED study. BMJ Open Diabetes Res Care 8(1). 10.1136/bmjdrc-2020-001506

284 [8] Bennet L, Nilsson C, Mansour-Aly D, Christensson A, Groop L, Ahlqvist E (2020)

285 Adult-onset diabetes in Middle Eastern immigrants to Sweden: Novel subgroups and diabetic 286 complications-The All New Diabetes in Scania cohort diabetic complications and ethnicity. Diabetes Metab Res Rev: e3419. 10.1002/dmrr.3419

[9] Bancks MP, Casanova R, Gregg EW, Bertoni AG (2019) Epidemiology of diabetes phenotypes and prevalent cardiovascular risk factors and diabetes complications in the National Health and Nutrition Examination Survey 2003-2014. Diabetes Res Clin Pract 158: 107915. 10.1016/j.diabres.2019.107915

[10] van der Heijden AA, Rauh SP, Dekker JM, et al. (2017) The Hoorn Diabetes Care System (DCS) cohort. A prospective cohort of persons with type 2 diabetes treated in primary care in the Netherlands. BMJ open 7(5): e015599

[11] Hebert HL, Shepherd B, Milburn K, et al. (2018) Cohort Profile: Genetics of Diabetes Audit and Research in Tayside Scotland (GoDARTS). Int J Epidemiol 47(2): 380-381j. 10.1093/ije/dyx 140

298 [12] Zhou K, Donnelly LA, Morris AD, et al. (2014) Clinical and genetic determinants of progression of type 2 diabetes: a DIRECT study. Diabetes Care 37(3): 718-724.

$300 \quad 10.2337 / \mathrm{dc} 13-1995$

301 [13] Yan M, Ye K (2007) Determining the number of clusters using the weighted gap 
medRxiv preprint doi: https://doi.org/10.1101/2020.12.21.20248628; this version posted December 22, 2020. The copyright holder for this preprint (which was not certified by peer review) is the author/funder, who has granted medRxiv a license to display the preprint in perpetuity.

It is made available under a CC-BY 4.0 International license .

\section{ACKNOWLEDGEMENTS}

306 This project has received funding from the Innovative Medicines Initiative 2 Joint

307 Undertaking under grant agreement No 115881 (RHAPSODY). This Joint Undertaking

308 receives support from the European Union's Horizon 2020 research and innovation

309 programme and EFPIA. This work is supported by the Swiss State Secretariat for Education,

310 Research and Innovation (SERI) under contract number 16.0097-2. The opinions expressed

311 and arguments employed herein do not necessarily reflect the official views of these funding

312 bodies. We acknowledge the support of the Health Informatics Centre, University of Dundee

313 for managing and supplying the anonymised data. ERP was supported by a Wellcome Trust

314 investigator award (102820/Z/13/Z). GAR was supported by a Wellcome Trust Senior

315 Investigator (WT098424AIA) and Investigator Award (212625/Z/18/Z), MRC Programme

316 grants (MR/R022259/1, MR/J0003042/1, MR/L020149/1) and by Diabetes UK

317 (BDA/11/0004210, BDA/15/0005275, BDA 16/0005485) project grants.

318

\section{AUTHOR CONTRIBUTIONS}

320 RCS, LAD, JWJB, LMTH, ERP designed the study and drafted the manuscript. RCS, LAD,

$321 \mathrm{HF}, \mathrm{GAB}, \mathrm{MA}$ performed the analyses. ID, DK, MI set up a federated node system for data-

322 analysis. RCS, DMA, LAD, HF, EA, AA, MJG, MK, FM, TS, AW, CLQ, MI were involved

323 in the data pre-processing and quality control. GNG, AF, MKH, DMA, IP, TJP, BT, VL, LG,

324 PWF, GAR, MJG, CK, KS, CLQ, AA, PR, AW, TS contributed to the data acquisition and

325 project logistics. All authors contributed to the data interpretation. All authors critically

326 revised the manuscript and approved the final version. RCS, LAD, JWJB, LMTH, ERP are

327 the guarantors of the work.

328

329 COMPETING INTERESTS

$330 \mathrm{KS}$ is CEO of Lipotype GmbH. KS and CK are shareholders of Lipotype GmbH. MJG is

331 employee of Lipotype GmbH. GAR has received grant funding and consultancy fees from

332 Sun Pharmaceuticals and Les Laboratoires Servier. MKH is an employee of Janssen Research

333 \& Development, LLC. AF and IP are employees of Eli Lilly Regional Operations GmbH. 
medRxiv preprint doi: https://doi.org/10.1101/2020.12.21.20248628; this version posted December 22, 2020. The copyright holder for this preprint (which was not certified by peer review) is the author/funder, who has granted medRxiv a license to display the preprint in perpetuity.

It is made available under a CC-BY 4.0 International license .

334 FIGURE LEGENDS

335

336 Figure 1 Characteristics of the clusters. a. Characteristics of the five clusters across three

337

cohorts DCS, GoDARTS and ANDIS. X-axis, cluster. Y-axis, age, BMI, HbA1c, HDL and

338 C-peptide b. Frequency and percentage of individuals in each of the clusters. SIDD, Severe

339 Insulin-Deficit Diabetes; SIRD, Severe Insulin-Resistant Diabetes cluster; MOD, Mild

340 Obesity-related Diabetes; MD, Mild diabetes; MDH, Mild diabetes with high HDL.

341

342

TABLES

343

344

345

Table 1 Characteristics of the included individuals of the three cohorts.

Variable

$\mathrm{N}$

Males, \%

Age, years

BMI, $\mathrm{kg} / \mathrm{m} 2$

$\mathrm{HbA} 1 \mathrm{c}, \mathrm{mmol} / \mathrm{mol}$

HbA1c, \%

C-peptide, nmol/L

HDL, mmol/L

LDL, mmol/L

Triacylglycerol, $\mathrm{mmol} / \mathrm{L}$

Glucose lowering, \%
DCS

2953

55.9

60.2[53.1-66.9]

29.5[26.7-33.2]

49.7[44.0-60.7]

$6.7[6.2-7.7]$

$1.0[0.8-1.4]$

1.2[0.97-1.37]

$3.0[2.3-3.7]$

$1.7[1.2-2.3]$

61.5

GoDARTS
5509
56.3
$62.5[54.5-70.0]$
$31.0[27.6-35.1]$
$58.0[50.0-79.0$
$7.5[6.7-9.4]$
$1.9[1.3-2.6]$
$1.1[1.0-1.4]$
$2.7[2.0-3.4]$
$2.3[1.6-3.3]$
19.0

GoDARTS

ANDIS

7478

60.1

62.0[54.0-69.8]

30.8[26.9-34.0]

62.3[45.0-74.0]

7.9[6.3-8.9]

1.3[0.9-1.5]

1.2[0.9-1.4]

$3.2[2.5-3.9]$

2.2[1.2-2.4]

59.6
347

348

349

350

351

352

353

354

355

356

357

358

359

\section{SUPPLEMENTARY FIGURES}

\section{Figure S1 GAP statistic and comparison to clusters based on HOMA measures a.}

Number of clusters versus the GAP statistic in DCS, GoDARTS and ANDIS. The most optimal number of clusters was at five clusters indicated by the dotted line. b. Comparison of clustering in ANDIS in the current study versus those identified in Ahlqvist et al.[1]. Numbers on the diagonal represent the absolute overlap (upper number) and the percentage (lower number). In the right panel, the sensitivity and specificity are given with $95 \%$ confidence intervals. SIDD, Severe Insulin-Deficit Diabetes; SIRD, Severe Insulin-Resistant Diabetes cluster; MOD, Mild Obesity-related Diabetes; MD, Mild diabetes; MDH, Mild diabetes with high HDL.

\section{Figure S2 All pairwise comparisons of identified clusters versus predicted clusters. Top} number in each cell represents the absolute overlap, the bottom number the percentage of 
medRxiv preprint doi: https://doi.org/10.1101/2020.12.21.20248628; this version posted December 22, 2020. The copyright holder for this preprint (which was not certified by peer review) is the author/funder, who has granted medRxiv a license to display the preprint in perpetuity.

It is made available under a CC-BY 4.0 International license.

360 individuals. Individuals were assigned to a cluster in cohort A based on the cluster centres of

361 cohort B and compared to the clusters identified in the cohort A. This resulted in the

362 following combinations: a clusters identified in DCS based on ANDIS centres $\mathbf{b}$ clusters

363 identified in ANDIS based on DCS centres $\mathbf{c}$ clusters identified in ANDIS based on

364 GoDARTS centres d clusters identified in GoDARTS based on ANDIS centres e clusters

365 identified in GoDARTS based on DCS centres $\mathbf{f}$ clusters identified in DCS based on

366 GoDARTS centres. SIDD, Severe Insulin-Deficit Diabetes; SIRD, Severe Insulin-Resistant

367 Diabetes cluster; MOD, Mild Obesity-related Diabetes; MD, Mild diabetes; MDH, Mild

368 diabetes with high HDL.

369

370 SUPPLEMENTARY TABLES

371

372 Table S1 Sensitivity and specificity of the cross-validation of clusters in other studies.

\begin{tabular}{|c|c|c|c|c|}
\hline Group & Sensitivity & Specificity & Cohort & Centers \\
\hline 1/SIDD & $96.22[94.98-97.23]$ & $98.62[98.3-98.89]$ & GoDARTS & ANDIS \\
\hline 1/SIDD & $97.06[94.8-98.52]$ & $3-97.91]$ & A & $\mathrm{D}$ \\
\hline 1/SIDD & $85.64[83.52-87.59]$ & $99.63[99.45-99.77]$ & DCS & ANDIS \\
\hline 1/SIDD & $88.78[86.61-90.72]$ & $98.59[98.21-9$ & ANDIS & GoDARTS \\
\hline 1/SIDD & $77.57[74.79-80.18]$ & $99.19[98.88-9$ & DCS & GoDARTS \\
\hline 1/SIDD & $97.06[94.8-98.52]$ & $96.36[95.56-9]$ & GoDARTS & DCS \\
\hline 2/SIRD & $92.29[90.05-94.15]$ & $92[91.33-92.64]$ & GoDARTS & ANDIS \\
\hline 2/SIRD & $36.05[32.32-3$ & $94.43[93.41-9$ & ANDIS & DCS \\
\hline 2/SIRD & $68.57[64.99-72]$ & $82.75[81.83-\S$ & DCS & ANDIS \\
\hline 2/SIRD & $56.19[53.02-59$. & $99.29[99-99.52]$ & ANDIS & GoDARTS \\
\hline 2/SIRD & $83.42[80.94-8$ & $89.67[88.75-90$. & DCS & GoDARTS \\
\hline 2/SIRD & 60.41-67.99] & $94.25[93.23-9$ & GoDARTS & DCS \\
\hline 3/MOD & 76.0 & $98.45[98.1-$ & GoDARTS & ANDIS \\
\hline 3/MOD & $73.85[69.84$ & $85.16[83.69$ & ANDIS & DCS \\
\hline 3/MOD & $54.96[52.58$ & $95.73[95.18$ & DCS & ANDIS \\
\hline 3/MOD & $93.21[91.53-94.65]$ & $92.72[91.91-93.46]$ & ANDIS & GoDARTS \\
\hline 3/MOD & $62.87[59.88-65$ & $96.27[95.67-9$ & DCS & GoDARTS \\
\hline 3/MOD & $74.23[70.24$ & $91.62[90.44-9$ & GoDARTS & DCS \\
\hline 4/MD & $66.15[64.3-67.96]$ & $91.53[90.71-$ & GoDARTS & ANDIS \\
\hline 4/MD & $51.04[47.65-54.42]$ & $74.46[72.53-76.32]$ & ANDIS & DCS \\
\hline 4/MD & $40.82[38.93-42.73]$ & $81.04[79.91-82.13]$ & DCS & ANDIS \\
\hline 4/MD & $78.14[75.95-80.21]$ & $83.13[81.93-84$ & ANDIS & GoDARTS \\
\hline 4/MD & $67.81[65.37-70.17]$ & $86.96[85.88-87.99]$ & DCS & GoDARTS \\
\hline 4/MD & $66.74[63.5-69.88]$ & $87.83[86.35-89.2]$ & GoDARTS & DCS \\
\hline $5 / \mathrm{MDH}$ & $92.75[91.15-94.13]$ & $94.18[93.57-94.74]$ & GoDARTS & ANDIS \\
\hline $5 / \mathrm{MDH}$ & $73.33[69.45-76.97]$ & $98.54[97.98-98.98]$ & ANDIS & DCS \\
\hline $5 / \mathrm{MDH}$ & $92.91[91.33-94.28]$ & $92.71[92.03-93.34]$ & DCS & ANDIS \\
\hline $5 / \mathrm{MDH}$ & $76.02[73.29-78.61]$ & $98.19[97.76-98.56]$ & ANDIS & GoDARTS \\
\hline $5 / \mathrm{MDH}$ & $89.47[87.43-91.28]$ & $96.36[95.77-96.89]$ & DCS & GoDARTS \\
\hline 5/MDH & $85.41[82.19-88.24]$ & $97.62[96.93-98.19]$ & GoDARTS & DCS \\
\hline
\end{tabular}


medRxiv preprint doi: https://doi.org/10.1101/2020.12.21.20248628; this version posted December 22, 2020. The copyright holder for this preprint (which was not certified by peer review) is the author/funder, who has granted medRxiv a license to display the preprint in perpetuity.

It is made available under a CC-BY 4.0 International license .

373

374 SIDD, Severe Insulin-Deficit Diabetes; SIRD, Severe Insulin-Resistant Diabetes cluster; MOD, Mild Obesity375 related Diabetes; $M D$, Mild diabetes; $M D H$, Mild diabetes with high HDL. 
HbA1c

HDL

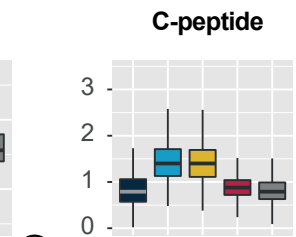

20 -



80 -

60 -

20 - 두붑 40 -
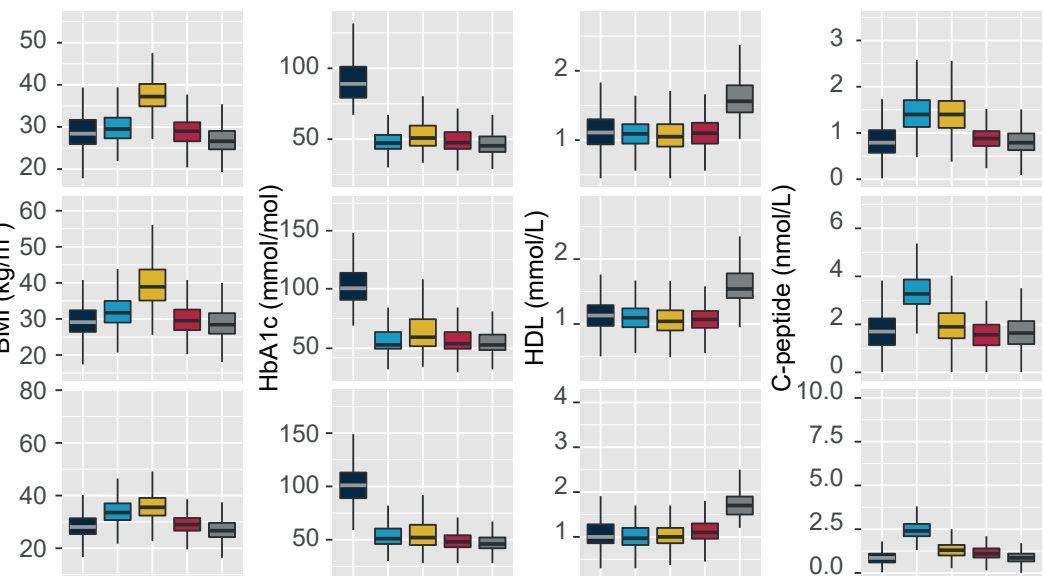


Cluster

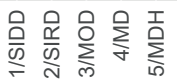

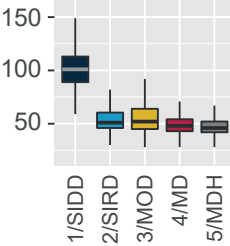

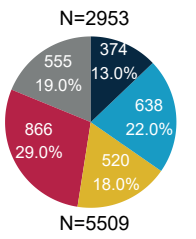

$1 / S I D D$

2/SIRD

$3 / \mathrm{MOD}$

$4 / \mathrm{MD}$

$5 / \mathrm{MDH}$
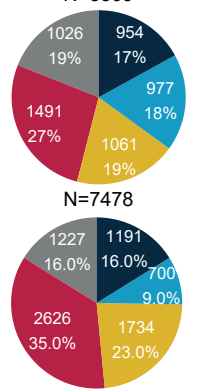

Figure 1 